Casa Nacional del Nifio.

Clinica Extraordinatia de Pediatria.

Prof. Dr. A. Ariztía.

\title{
LA CONSTITUCION EN SUS RELACIONES CON EL PROCESO TUBERCULOSO
}

Por el Dr. FEDERICO EGGERS.

Tarea difícil, por no decir vana pretensión, encierra el título de la presente exposición que he terido que ceder a las insistencias de nuestro distinguido Presidente, al traer esta colaboración un tanto vaga todavía y confusa por la dificultad y normas estadísticas rigurosas.

Ha pasado con la Tuberculosis, lo que no ha pasado con enfermedades que comprometen el metabolismo, en las cuales se estudiaba el organismo en su totalidad. La Tbc., como enfermedad infecciosa y su variabilidad individual, se explicaba por la frecuencia y masivídad de la infección, virulencia bacilar, medio ambiente, clima, que eran responsables en último térmimino y los que justificaban a su vez, su polícroma modalidad individual. Ha pasado, y todavía se mantiene en nuestras mentes dicha creencia, creencia unilateral pero explicable en medicina en sus esfuerzos de perfección. En pediatría pređominó en los trastornos nutritivos o enfermedades nutritivas, ese criterio. Basta recordar la sucinta bistoria que de ellos realiza el Prof. Czerny al hablar de la pediatría de su tiempo, quien no recuerda la era bacteriológica de las infecciones gastrointestinales, el microbio, el intestino, la importancia monosintomática. sin considerar el conjunto, el terreno, en el cual se desarrollan y desenvuelven. Vienen después las estadísticas; con ellas las dudas, y se plantea el problema: ¿Por qué en presencia de un contagio general, sólo un pequeño tanto por ciento enferma de tuberculosis? ¿A dónde radica la resistencia, por ejemplo, de la juventud escolar, con una mortalidad de $1,9 \times 10.000$. contra el $9 \times 10.000$ del adulto? ¿Por qué en el niño la ptisis 
pulmonar es sólo un $3 \%$ de là Tbc. pulmonar? ¿Por qué en Ellos es más frecuente otras formas de Tbc.? Todo esto condujo a pensar en sus fuerzas internas, en su constitución, que, sin duda, interviene rn la evolución de la tuberculosis.

Ahora bien, para comprender las relaciones individuales de una contextura, frente al proceso tuberculoso, necesitamos exponer el concepto constitucional y sus modalidades más importantes. La contextura individual diversa, abarca desde luego, a todo el ser, desde su aspecto externo, el hábito, a su comportamiento fisiológico y funcional (reacción al estímulo); a su actitud o comportamiento psíquico, etc., etc.; su reaccionabilidad representada por su fenotipo, es decir, la apariencia o aspecto en conjunto de un ser humano, desde el momento que nos comenzamos a preocupar de él. El ser humano se compone de dos factores: $1 . \because$ la masa heredada (constitución heredada) y 2.․ las influencias que el medio ambiente pueda ejercer sobre ella. En ello el Geno es lo más importante, determinante y constante, lo inmutable; pero por influencias externas o medio, comportarse diferentemente en su fenotipo. Se comprende que esta influencia peristática de la constitución, es leve. y sólo lo que autoriza individualmente su Geno. Pero alcanza a ser lo suficientemente grande para lograr terapéuticamente una justificación en cierto sentido de transformar o influenciar la constitución (disminuir, por ejemplo, una altetación patológica exagerada de los tejidos a estímulos externos).

En los comienzos, se atribuyó gran importancia a los caracteres externos de la extructura orgánica, su morfología, se pensó que las formas externas, el hábito, sería lo primordial como expresión de la constitución (Hábitos de Sigaud) hoy reducidos a tres tipos: $19^{\circ}$ Muscular: $2 .^{\circ}$ Digestivo o pícnico: 3. Leptosómico, que resume al Asténico, Respiratorio y Cerebral: hubo necesidad de extender los estudios hacia el comportamiento reaccional y psíquico dados por su patrimonio herencial o caracteres dados por la raza, por dicho motivo, lo principal dentro del marco de la constitución. es la respuesta a un estímulo o reacción funcional, sin de jar de tomar debida consideración a los otros factores de la constitución. Y es así cómo el modo de reaccionar de un ser humano, es la llave de su comportamiento corporal durante un período, sea de enfermedad o de salud, y para la comprensión médica, representa el factor más importante de la constitución.

Pues bien, la diferencia constitucional en la reacción a los estímulos, se manifiesta en la vida diaria: resistencia, cansancio, sensibilidad al dolor, a los cambios atmosféricos, tolerancia por 
el frío o calor, acción del sol, etc. De ahí nace, además, una de sus peculiaridades: "su variabilidad", parą su comprensión, es requisito abatcarla en su conjunto, para poder juzgar las variaciones que puedan tener de lo conceptuado como normal. Por eso la Constitución no se deja ni se puede enseñar como una al. teración patológica determinada como pasa con el sarampión, la neumonia o el tifus; requiete de los fundamentos científicos asociada a una sensibilidad médica adquirida con la experiencia. Sólo así se puede compenetrar o percibir la extructura funcional de un ser humano. su reaccionabilidad, su comportamiento frente a un estímulo, relegando a segundo plano, las medicioné: y cifras ponderales que sólo abarcan lo morfológico.

El organismo puede variar en su modo de ser orgánico. basta recordar las perturbaciones funcionales de la Titoides, Hipófisis, Suprartenal, etc. que conducen en último término, a estados patológicos manifiestos. Menos conocidos y de mayor importancia para la práctica diaria, son las alteraciones o desviaciones que a los estímulos experimenta el sistema mesenquimático. Según su grado o intensid-d, se describen los Hiper. Normo o Hipomesenquimáticos. El primero comprende la constitución excitable que encierra al exudativo, al diátesico neuropático, el neurolinfatismo, la espasmofilia, la vagotonía. la diatesis alérgica. AI hipomesenquimático lo forman la astenia universal congénita. No se trata de estados corporales precisos - bien determinados, sino sa diferencia radica en una mayor o menos excitabilidad o itritabilidad en el sentido de una hipo.o hiperfunción de sus tejidos.

La constitución irritable, con su exquisita sensibilidad de piel y mucosas, sus órganos linfáticos, etc. comprende los indi. viduos predispuestos a adquirir infecciones banales, perturbaciones en el sueño, decaimiento matutino, tandencia a las alzas febriles indeterminadas, perturbaciones vasomotoras, asma, urticaria, neurodermitis, etc. El aspecto externo de estos niños exudativo-linfáticos, permite una diferenciación: el flaco y el pastoso caya descripción no detallamos por la brevedad del trabajo y porque basta consultar los textos respectivos.

Funcionalmente diferente a la anterior, está la Astenia. que no representa un estado orgánico, sino una función corporal, es decir, debilidad funcional de la musculatura y tejido conjuntivo. de los sistemas que dan ese cuadro caracterizado por tórax paralítico, costillas verticales, ángulo epigástrico agudo. escápulas caidas, descenso diafragmático, corazón en gota, ptósis visceral, mala formación de anticuerpos y neoformación de tejidos. Por no desempeñar un rol determinado en la infancia. 
no nos preocupará por ahora. Aparece después de los 10 años y alcanza su máximum entre los 15 y 30 años.

Si el concepto de la constitución es importante en el individuo sano, mayor es en el organismo enfermo. Su naturaleza influencia el curso y evolución de una tuberculosis. La infección Tbc. con su contagio tan general, que sólo en un pequeño tanto por ciento conduce a la enfermedad o a la muerte. Sin embargo, la evolución y multiplicidad de sus cuadros clínicos. da margen a pensar en la individualidad de cada caso, no obs. tante el hecho de observarse grupos uniformes. La primoinfec. ción en el lactante y niño pequeño o en edad más tardia, pueda evolucionar en forma inaparente. La positividad de la reacción a la tuberculina, indica la infección. Junto a estas formas se observan Tbc. ganglionares de los hilios. infiltrados primarios y neumonia caseosa. Mientras más joven es el enfermo, mayor posibilidad de que se llegue a la enfermedad tuberculosa. La neumonia caseosa concomitante a la primoinfección, pertenece casi exclusivamente al lactante. En el pre-escolar, además de las formas descritas, aparecen manifestaciones secundarias, Tbc. quirúrgicas. Esas formas vuelven a encontrarse en la edad del escolar aumentando el porcentaje de las Tbe. de diseninación, o al íniciarse el desarrollo, el infiltrado precoz con otras ptisis de la pubertad propiamente dichas. En el adul. to, desaparecen las Tbe. extrapulmonares, predominando la ptisis pulmonar.

¿Cómo explicar ese polimorfismo del proceso $T b c . ?$ ¿A qué se debe la escasa mortalidad y enfermedad en el niño pequeño y escolar? La inmunidad específica dada por la primoinfección. no basta para explicarla. Sin duda la reacción in. dividual dada por la constitución. parece oponerse o favorecer la evolución del proceso; pero aún la esquematización de los cuadros como lo realizó Ranke, no puede contestar el por qué tal niño padece de una infiltración o cual fallece de una meningitis Tbc.. ¿Por qué la niña $X$ padece de una ptisis galopante y mortal y la $Y$ una Tbc. que rápidamente alcanza su curación. o por qué algunos no llegan a enfermar de Tbc.? Tal vez el conocimiento de una contextura orgánica expresada en la respuesta que pueda dar a diferentes estímulos, pueda ser Ia senda que nos conduzca a un más allá en la evolución del proceso mismo.

La constitución de un hombre es algo absoluto; se revela en forma de una teacción precisa, bien determinada a estímulos exógenos o endógenos. La Disposición es algo relativo; es la predisposición o facultad que posee un individuo a contraer 
tal o cual enfermedad. La disposición depende de la constitución. Investigaciones practicadas en los gemelos, ha podido demostrar como hecho probado, de que existe una disposición a la Tbc. Además, es o no la disposición a la tuberculosis, una predisposición constitucional independiente o dependiente de otras anomalías constitucionales conocidas y ser ellas su causa en último término? Este problema es el que ocupa mayor in. terés en la actualidad. Se pensó primero que el hábito podria expresar una contextura orgánica: sin embargo, los resultados obtenidos con el compás y la huincha, no dejaron traslucir la constitución Tbc., a pesar de que los leptosómicos son frecuentes entre los ptísicos. Hoy se rechaza para el adulto, la existencia de estigmas constitucionales externos que puedan predisponer a la $\mathrm{Tbc}$. Igual pasa en los niños. Pero las relaciones son diferentes al considerar o analizar la manera de reaccionar del organismo. frente al proceso Tbc., y éste es el problema básico que nos ocupa.

Là Astenia, es decir, la alteración constitucional con una menor reacción a los estímulos, puede actuar desfavorablemente en la evolución de un proceso tuberculoso. Es sostenida $\mathrm{y}$ mantenida por muchos autores. Se deberia en último término. a su modo de ser. ss decir, a una disminuida capacidad de reacción en general $y$ en especial, a una ausencia de proliferación reaccional conjuntival o insuficiencia de producción de anticuerpos. Algunos autores encuentran una disminución de las lipasas sanguíneas.

El problema constitucional en la tubetculosis, adquiere su verdadera impurtancia desde el momento en que se inicia la lucha Asistencial Antituberculosa en que se remiten a los asilos y policlínicas a niños predispuestos con pseudoadenopatías, a los rotulados como inferiores constitucionales. a niños débiles, es decir, a exudativo-linfáticos. Se vió precisamente que éstos niños no padecian de la enfermedad. La importancia del linfatismo en la Tbc. encuentra su aprobación en la literatura. Pfaundler lo considera junto con Czerny, como un factor de pronóstico favorable en el curso de una Tbc. Müllet habla de una resistencia que le es conferida por ella. Kleinschmidt encuentra un alto tanto por ciento de linfáticos, en las formas quirúrgicas de curso benigno. Schultz habla de una mayor resistencia frente al bacilo de Koch. Borchardt habla de mayor resistencia y mejor pronóstico en los linfáticos con Tbe. pulmonar comparada con el asténico y normalmente constituído debido a la mayor facultad de reacción de sus te. jidos linfáticos. Schlack habla de la benignidad de los infil- 
trados pulmonares e insiste en que la mayor parte de los pacientes, corresponde a los que ofrecen estigmas de constítución exudativo-linfática y hoy se llega hablar en ellas, de una escrofulosis del pulmón.

Según Brandt, las escasas ciftas de mortalidad de la Tbc. en la infancia, entre 2 y 15 años, se deberia en último término, a la hiperplasia linfática. Además, los elementos constitutivos del pulmón infantil, no hacen más que ofrecer circunstancias óptimas de defensa: buen desarrollo bronquiolo alveular, tejido conjuntivo blando y suelto, elasticidad y ganglios linfáticos bien desarrollados, relaciones que faltan mu. chas veces en el adulto por procesos de involución o atrofia (además precoz en el asténico, comparado con el pícnico).

Son frecuentes y numéricamente superiores los niños con manifestaciones exudativo-linfáticas. en algunos puede ptedominar la componente exudativa, en otros la linfática.

Todo esto no quiere decir de que existan excenciones. que linfáticos puedar sucumbir, como sucumben, de Tbc:. 0 , a la inversa. Pero al analizar un problema en su conjunto, no deben cegarse ante las excepciones. Pero la observación cuidadosa, prolongada y bier estudiada, por lo menos indica la rareza de la ptisis en el línfático. La Tbc. abierta es menos frecuente en el linfático, es más favorable compatada con el que no lo es.

Todo esto conduce a un resultado práctico: Considerar cada caso en su conjunto y no autoriza arriesgar o a practicar una terapéutica o actos quirúrgicos, sin pensar previamente que existe un factor en pro o en contra, es decir. la constitución.

Se abre una eta, la era de la reacción individual frente a un agente infeccioso, sea específico o banal. También abre una brecha de incalculables proyecciones en el terreno de la Patología del Lactante. La clinica debe ser la clinica reaccional, funcional, propia en cada caso. Recuerdo las palabras pronunciadas por uno de los maestros de nuestra especialidad que al dirigitse a las futuras generaciones pediátricas, decía: El médico que para las anorexias sólo conoce los eupépticos o el cirujano que después de practicar una elegante incisión en un absceso o antrax, dan, junto con exprimir la última gota de pus o su firma ante una magna receta, como terminada su misión, muy lejos están de la verdadera posición que les corresponde adoptar. Pensar en una pertutbación funcional de causa múltiple, en uno, o de. una mala inmunidad en otro, causas dignas de investigar en cada caso. 
Para terminar, ruego excusas y agradezco la paciencia aue han tenido en escuchar esta modesta y desordenada presentación que indica por lo menos. el gran interés que nos debe metecer el problema de la constitución en la evolución del proceso tuberculoso.

\section{tran la disertación. \\ A continuación una reseña de los casos clínicos que ilus.}

1.-OBSERVACION $7164 \ldots$ J. B. Naido el 10 di ago:to de 1936. Ingresa de 5 meses de edad al dia 7 de encro de 1937. Diátesis exudativo-linfática. Hijo de madre Tber- Fué alimentado y convivió con ella tres meses. Ingresa con un peso de 5 kilógramos. Hermano mayor de 4 años, tuberculose. Presenta rinofaringitis a repetición, abscesos, piodermits y procesos suputativas disecantes que forman un verdadero manto que cubre todo el dorso. Tu. betculinas (-). A los nueve meses se hasen positivas. Radioscopias y radiografias sucesivas, sólo indican ana sombea de infilteación periganglionar inferior derecha. Examen de desgatro positivo en dos ocasiones. Inoculatión positiva. Presenta además varias fistulas isquio-rectales rebeldes, con abundante sapuración que se catalogan como tuberculosas. Llega al aío de edad en pr'catias condiciones nutritivas, catalogado como una dexcomposición con $3.500 \mathrm{grs}$ de peso. Tratado con tansfusiones sanguineas \& LH. mejota su estado genetal. Disaparecen sns lesiones catáncas $f$ supurativas de los ganglios submaxiJares, no así las iqquí-rectales. Mzjora su estado general, $\gamma$ al año y medio pesa $6.500 \mathrm{grs}$. Pirquet positivo. Radiográtia pulmonat positiva, Uleeraciones escrofolosas de la cómea. Tubercuides. Xlcanza los 2 años de edad. $y$ pesa $9 \mathrm{~kg}:$. A los 2 años 8 meses. pesa $12 \mathrm{~kg}$. Presenta una conjuntivitis fliitenular bilateral. Radiografia pulmonar i.-.). Tuberculinas positivas. Persisten afecciones catarrales bronquiales $y$ lesiones ganglionares múltip'es supurativas. Hoy esta en colocación familiar. Hizo también un satampión del saal convalece sin acusat mayores alteraciones.

Comentario. - Niño tuberculoso con caracteristicas y hábito exudativo-linfático que alcanza la descomposición y que mejora y resiste la evolución de su proceso tuberculoso.

2.-OBS. 8494,-H, G. Hijo de midre con Tb. exudativo bilatera! cavitara con varias hemoptisis. Tuvo 6 embarayos: 5 pattos de término $y$ i aborto, 3 bijos vivos. 2 fallacidos, 1 de meningiris Tbe. Los hermanos mayores con tubercalinas positivas y examen pulmonar (--l. Estuvo siempre en contacto con su madre snferms. alimentado exclusivamente por ella basta los 4 meses: fecha en que ingresa a la $C . N$. del $N$, con un peso de 6.100 ges. Diagnótico: Diátesis exudativa. Hábito pastoso. Alimentada attificialmente, progresa en buena forma. Tubarculinas hasta concentración I $x 1000$ (一). A log 7 y medio meses pesa $9 \mathrm{kgs}$. Coríza. Queratocorjuntivitis flictenular. Pirquet (-). No así Magtoux que fué positivo al $1 \times 1000$. Eirqust 
2 los 9 meses positivo ( 3 cruces). Radiografia: Pequeña sombra peribiliar inferior derecha. Sedimentación: $26 \mathrm{~mm}$. Régimen sin keche. Jo wlera bien. AI mes. sedimentación $16 \mathrm{~mm}$. y con dicho ségimen llega al año de edad can un peso de 11.400 grs., sedimentación $13 \mathrm{~mm}$. En esa fecha se instala anorxia, palidez, fiebte, irtitabilidad, somnolencia, recidiva de su conjuntivitis flictenular, tuberculides numero:as y bien aprecizbles en ponssées sucesivas. Sedimentación $45 \mathrm{~mm}$. Todo evoluciona en 6 semanas, recupera sa apetito $y$ buen bumor. Sale de alta con Piogurt débilmente positivo. Sedimentación 15 mm. Radiografia pulmonar sólo una secuela con tendencia a la calcifitición. Peso: $13 \mathrm{kgs}$. Sale a los 17 meses al sarvicio de colocación familiar. Astualmente está en Apoquindo $\mathrm{Y}$ su cuidadora lo trae periodicamente al Se:vicio.

Comentârio.-Exudativo linfático. Tuberculoso. Diseminaciones a repetición y evolución favorable de su Tbc.

3.--OBS. 8174.--M. G. Ingresa por orden judicial en agosto de 1937. más o menos a los 2 meses de edad. Diátesis neuropática. Irritabilidad. Vomitadior y ragungitador habitual. Caprichoso para comer. Anorexias nerviosas. Mfecciones cutáneas intertriginosas. Después de los 6 meses, bronquitis assática a repetición. Corizzs. Sale de alta a los 9 meses con $8 \mathrm{kgs}$. de peso y tuberculinas y radiografias (-). Reingresa al año, es decir, a los 3 meses de estadía fuera del Asilo con 6.900 grs. de peso. Distrofia avanzada. Pirquet positivo ( 3 cruces). Radiografía pulmonar da: Sombra de infiltración extinsa en la base inquierda con sintomas elínicos de condensación. No tiene alzas termicas. Larga evolución sin repercusión en su escado general. Aparece eczemi do toda la cara. mejilla y frente con impetiginización secundaria. El estado pulmonar persiste sin variación hasta los 17 meses, fecba en que contrae unit escarlatina complitada de adenitis supurada febril. A los 18 meses cu tivo del lavado gástrico por concintración e inoculación experimental positivos. Secí. mentación $12 \mathrm{~mm}$. Sombea de condensación prersiste igual. Coqueluche sin mo. dificar su proceso Tbe. Aleagza a los 2 años de edad con ur peso de 12,500 ges. Su proceso pulmonat desaparece dejando como secuela un foco calcillew. do. Su lesión eczematosa, rebelde busta entonces, ezdz con un régimen sin leche, mejota su caracter en forma visib's.

Comentario. - Lactante con diátesis neuro-exudativa. Tuberculoso que evoluciona favorablemente a pesar de pre. sentar dos enfermedades gtaves intercurtentes.

4.--E. G. Ingtesa de un año de edad. Diatesis exadativo-linfática. Halito pastoso (escrofaloso). Había estado hospitaizado ent el Hospital Arra. rán dande se diagnosticó un complejo prımąio izquierdo con infíltración ptrifocal extensa quz ragtesa casi en su totalidad. Fistulas perianales tuberculosas. Tubercolinas intensamedte positivas (con 3 crufes). Inoculación experimental 
sositiva. Su estado general se conserva en buends condiciones hasta 1 anio 11 meses de edad. Lesiones puimonares en regresión casi completa. Sedimentacion 'O mm. por hora. Contrae una toqueluch: complreada. Eebril. Sedimedtación y. mm. Conjuntivitis flictenular. Tuberculides. En el etrso de su coqueiuche. arampión complicado de bronconenmonia doble, sarampionosa gave. El Pirquet se hace (-) y a los 15 dias de iniciado su sarampión se hace positivo. Se thasra una agravación manifiesta, se instala ana disemiatión pulmonar. sin mbargo. el extado general mejora, se normaliza su temperatura, Sedimentacion $14 \mathrm{~mm}$. Poiteriomente nueva bronconeumonia que evoluciona en tres senianas sin repercutir sobre su proseso Toc. Hoy día está en buenas condiciones di alud. en franca convalecencia.

Comentario. - Diätesis exudativa-linfática. Tabercuiosis. Diseminación y mejoría a pesar de presentar varias en. tormedades graves intercurrentes.

5.-G. B. C. Ingresa a los 11 meses de edad. Su comportamiento es ac una niña neuropática. Constitación excitabie. Labilidad nerviosa. Madre Tbi. convive con ella durante 7 meses. Pirquet intensamente positrvo (3 craces). Kadiografía pulmonar: Sombra de infiltración homogénea que ocupa totio $e$ : tcrcio superior del campo pulmonar derecho. Adenopatía bilatezal. Es víctima de rinofaringitis, grippe, pénfigo. abscesos múltiples de la pial a repetición ợ- fsiva de afecciones grippales. A pesar de todo. sus lesiones específicas tienden a revelar una franca regresión, calcificación aún intrapulmonares, A $\operatorname{los} 2$ años rée pdad pesa $10 \mathrm{kgs.}$ franca regresión de sa proceso pulmonat. Se me olvidab., recir que lo inoculación experimental fué positiva. A los 2 años 7 meses dz pilad. eccarlatina grave complicada; teaparición de su infiltración en el pulmö́n trrecho. Después coqueluché y sarampión atenuados (hemoterapia preventiva). birr repercusión alguna sobre su ptoceso tabetculoso. Hoy tiéne 3 años 5 meves ie edad. Pesa 12,750 grs. Examen pulmonar (-). Una prueba de tubercu. lina persiste po:itiva por espacio de 22 dias. Sedimentación 9 mm. Fiátula de s: adenitis supurada del cuello en regresión.

Comentario. - Diátesis neuropática. Constitución exc!table. Labilidad neurovegetativa. Tuberculosis. Enfermeda. des intercurrentes graves. Evolución de su Tbc. hacia la mejoría.

6.-OBS. 7837. J. M. C. Ingtesa de 14 meses de edad con un pese de $7.800 \mathrm{grs}$. Hijo de madre tuberculosa cavitaría y bacilifera. alimentado por e:lu hasta los 10 meses de edad. I.a madre fa'tece un nes deipués de su ing:cio. Pit¡uet y Martoux (-). Lesiones de la piel eczematizadas. Sarna. Coriza. Hábito pastoso. Diátesis exudativa. Situs inversus. Salz a colocación familiar. Dermutitis seborreica en los pliegues $y$ enero cabelludo impetiginizada secun- 
Jariamente, rebelde y prürigo que lo bacen reingresar. Eczema de la cara. Ein tl servicio, gloméru'onefritis. Aecciones cutáneas recidivantes que obligan a un régimen sin leche. Se administra por la rebeldía de su afección del caero cabelludo, acerato de talio. Tuberculinas y exámenes radiolópicos (-), A los ? 2ños 10 meses pesa $13 \mathrm{~kg}$.

Comentario. - No contrae infección Tbc. a pesar de estar expuesto a un posible contagio. Habrá herencia disposicional desfavorable a la infección Tbe?

7.-OBS. 6286. J. B. A. Hijo de madre Tbe, que acude en seguida al Hospital San Jo:é con una Tbc. bilateral exudativa y tuberculosis laringea grave. Afónica y abundantes $B$. K. en su desgarro. Fallece a los pocos días de hospitalizada. Alimentado exc'asivamente por ella durante un mes y mixu hasta su ingreso a los 7 meszs con $7,150 \mathrm{kgs}$. Eutrófica. Hábito linfático. ['Pre. :untó en el servicio bronquitis asmática a repetición y tébeldes a tojo tratamiento. Tuberculinas hasta concentraciones de l $x 10(-)$ a repetición y periódicas. Examen de rayos (-). Fallece a raíz de una coqueluzbe complied. tia de bronconeumonia a los 18 meses de edad. Autopsia: Realizada minuriosa. mente por el Dr. Guzmán, dice: No se eonstatan lesiones tuberculosas de nizguna especie.

Comentario, - No contrae ni padece de la afecciọn tuberculosa. Existe una constitución refractaria a la tuberculosis?

8.—OBS. 9062.-3. C. Niño de 3 años 2 meses. Peco 15 kgs. Padro rallecido de Tbc. Examen pulmonar (-). Pirquet positivo (3 cruces). Mo'de -a cera del Pirquet positivo practicado a los 10 días. Escrofulosis. Querato- ngjuntivitis flictenalar. Residuos y secuelas de sus características constitucionales en la piel. B:onquitis tecidioantes y coriza crónico.

Comentario.--Será la escrofulosis la expresión máxima de la constitución exudativo linfática y su gran alergia la expresión de su máxima defensa?

9.-S. C. Hábito exudativo-linfático. Tuberculinas (-). Catacteristicas de su reaccionabilidad extracordinaria a enfermedades catarrales de las vias re:firatorias y atributos propios de su constitución.

Comentario.- Se tratará de modificar o ensayar en é! alguna acción peristática de la constitución, es decir, su fenotipo, dentro de las variaciones que permita su genotipo?

10.-E. E. Nacida el 3 de agosto de 1936. Ingresa a los pocos días a ta C. N. N. Prematro, sio antecedentes de Tbc. Dermatitis soborreica. Coriza. 
Comportamiento reaccional como una diatésico exudativo discnero. Tubercentí. nas (-). En fébrero del 39, que:atitis ulcerosa ojo izquierdo, E1 14 de diciembre de 1937, examen radiológico y tuberculinas (-). Febtero del 39. Pirquet positivo, razón que obliga a hospitalizarse en el Artiarán. Radiografto en aquel entonces: Complejo prinatio que evoluciona sin complicación $y$ es dado de alta en buenas condiciones. Sólo persiste su queratitis. Vuelve a is 4. N. N. Su queratitis es rebelde. Su examen pulmonar es (-).

Comentario,-Niño exudativo-linfútico exento de ttlberculosis que contrae su afección tuberculosa. Adquiere por su reaccionabilidad constitucional, los atributos de un escrotuloso, la expresión máxima de una diátesis exudativa?

11.-E. L. Fué alimentada al seno materno basta un año de edad. Ls uadre fallece de una Thc. exudativa bilateral. Ingresa a los 3 años y meses de edad con Pirquet positico (2 cruces) y sombra de infiltración pu'monar perthiliar detecba (infiltración periganglionar).

Comentario. - Neuropacia constitucional. Irritable. Emotividad. Terrores nocturnos. Reaccionabilidad del terre. no a la infección? 